\title{
An intravenous biological therapy for psoriasis: Infliximab
}

\author{
M. Mathur ${ }^{1}$, S. K. Kedia ${ }^{2}$, R.B.K. Ghimire ${ }^{3}$ \\ ${ }^{1}$ Professor, ${ }^{2}$ Senior Resident, ${ }^{3}$ Junior Resident; Department of Dermatology, College of Medical Sciences, Bharatpur, Chitwan \\ District, Nepal.
}

\begin{abstract}
Psoriasis is a common, chronic inflammatory skin disease which typically follows a relapsing and remitting course and is associated with joint disease. The significant reduction in quality of life and the psychosocial disability suffered by patients underline the need for prompt, effective treatment and long-term disease control. Patients with moderate to severe disease often require systemic treatment with effective \& new modalities such as biological-Infliximab.
\end{abstract}

Review will highlight fundamental aspects of infliximab and its use in Psoriasis as well as provide specific comments regarding this monoclonal antibody and its position in of psoriasis in the future treatment.

Key words: Infliximab, monoclonal antibody, dex, quality of life.

\section{Introduction}

Historically, Psoriasis is not a newly discovered skin disease. Although advances have been made in the management of the disease but much of the stigma of Psoriasis still persists. It is estimated that nearly $2 \%$ of the global population suffers from Psoriasis. ${ }^{1}$ Psoriasis does not discriminate between gender and all ages can be affected.

Psoriasis is a common, chronic immune-mediated disease typically characterized by skin lesions that are erythematous scaly plaques and may be associated with joint disease in approximately $25 \%$ of patients. ${ }^{1}$ Psoriasis can have a polymorphous presentation, and may vary in severity from patients

Correspondences: M. Mathur

E-mail: maheshmathur2@hotmail.com to patients. Classically, a patient would present with asymptomatic erythematous plaques concentrated on the extensors of body, palms, soles, scalp and may involve nail. At times, manifests as erythroderma.

The significant reduction in quality of life is being reported and is in parallel with the other medical diseases such as Diabetes, Rheumatoid arthritis. Regardless of the severity, Psoriasis has a dramatic effect on a patient's health-related quality of life (HRQoL) and overall quality of life (QoL) influencing their career, activities, family and virtually all other aspects of their life. ${ }^{2,3}$ Psoriasis is an unpredictable disease. The patient may have a single episode followed by clearance and no recurrence where as another patient may have disabling, life-long disease with arthropathy. Most 
patient experiences periods of flare and remission leading to frustration for both patients as well as treating physicians. The psychosocial disability suffered by patients necessitates urgent need for effective treatment and long-term disease control. However, localized limited disease can be managed satisfactorily with topical agents but patients with moderate to severe disease often require systemic treatment.

\section{Management}

The management of moderate-to-severe Psoriasis has changed dramatically and burdensome inpatient treatment of Psoriasis is no longer a common practice. Psoriasis now can be managed in outpatients, though decision making for the treatment by physician remains complex because of comorbidities, toxicity, accessibility and efficacy of therapy along with patient preference. ${ }^{4,5}$ A classic approach to Psoriasis treatment is stepwise, beginning with the least invasive and safest treatment followed by more potent and potentially more risky therapeutic options until disease control is achieved. ${ }^{6,7}$ Efficacy of therapeutic modalities in the treatment of Psoriasis is frequently measured as the percentage of patients who achieve $75 \%$ improvement in the Psoriasis Area and Severity Index (PASI75).

In the last several years, our understanding about immunopathologic mechanisms of Psoriasis has advanced tremendously and therapeutic agents have been designed to interrupt key pathways in the chronic immune inflammation at molecular level.
The most recent additions in the therapeutic armamentaria are biological agents in the form of Monoclonal antibodies. Biologic therapies are designed to mimic certain 'naturally' occurring proteins that when used therapeutically, interrupt signaling of the immune system and at times, induce death of the target cell. They represent proteins that are produced using recombinant DNA techniques.

Current biological therapies approved for the treatment of Psoriasis include alefacept, efalizumab, etanercept and infliximab. Adalimumab - tumor necrosis factor-alpha (TNFá) antagonist reported to have impressive efficacy in Psoriasis. The comparative study of the biological agents showed that each has its advantages and disadvantages and no single biologic is right for every patient. ${ }^{8}$ However, they have shown good efficacy in clinical trials and rapid therapeutic response providing significant improvement in QoL in moderate to severe Psoriasis patients.

Infliximab is a unique biologic therapy in the treatment of Psoriasis as it is the only intravenous TNF-á antagonist with every other month administration. It has some of the most impressive efficacy data and is well tolerated. Infliximab therapy provides patients with a two month holiday from systemic therapy because of the typical dosing regimen. Regardless of the utility of more traditional conventional therapies, such as methotrexate, acitretin and cyclosporin, infliximab and other biologics are useful in Psoriasis treatment 
because they provide safe and effective alternative. Infliximab fits into the Psoriasis treatment paradigm.

Pharmacodynamics and Pharmacokinetics of infliximab-TNF-á levels are reported to be elevated in Psoriatic plaques which support the rationale of using infliximab and other TNF-á antagonist in Psoriasis. Infliximab is a chimeric (derived from human and nonhuman gene sequences) monoclonal IgG antibody that binds TNF-á, a potent inflammatory mediator. The variable portion of the antibody is coded by sequences common to both human and murine DNA. Infliximab binds soluble TNF-á which is already bound to its receptor. ${ }^{9}$ Infliximab inhibits the inflammatory response by blocking TNF-á that leads to decrease in the proinflammatory cytokines propagating chronic inflammation. It has been observed that in Psoriasis plaques, the levels of TNF-á, as well as the numbers of $\mathrm{T}$ cells are reduced following infliximab infusion. ${ }^{10}$

Infliximab is supplied as $100 \mathrm{mg}$ of preservativefree powder that is reconstituted prior to intravenous infusion. Dosing is weight based, typically $5 \mathrm{mg} / \mathrm{kg}$ given intravenously initially, followed by dosing at 2 and 6 weeks, and every 8 weeks thereafter. At steady- state kinetics, the half-life of infliximab is 7-10 days. Anti-infliximab antibodies developed in $27-47 \%$ of infliximab-treated patients. These antidrug antibodies correlated with decreased serum infliximab levels and increase in dose in standard regimens. However, the concomitant use of methotrexate may prevent the development of these anti-infliximab antibodies. Patients receiving infliximab for Psoriasis have developed antinuclear antibodies (ANA), as well as anti-dsDNA antibodies. The clinical significance of these antibodies is unknown but the development of lupus-like syndromes has been reported in patients treated with infliximab. However, resolution of such lupus-like syndromes is the rule with cessation of therapy. ${ }^{11}$

There are three pivotal randomized, double-blinded, placebo-controlled trials that demonstrate the efficacy of infliximab in treating plaque-type Psoriasis. Clinical trial of 378 moderate-to-severe Psoriasis patients receiving infliximab, $5 \mathrm{mg} / \mathrm{kg}$ at weeks 0,2 and 6 showed an $80 \%$ PASI 75 rate at week $10 .^{12}$ The other two studies show PASI 75 response rates of approximately 70\%. The onset of response with infliximab is very rapid. Patients frequently report significant improvement after only one dose. Mab is of value in patients who like relatively long holidays between infusions and in those patients who has poor adherence to therapy.

Clinical trials are typically assessing efficacy and common adverse events. The events reported since the original clinical trials are - neutropenia, interstitial pneumonitis, idiopathic thrombocytopenic purpura, systemic and cutaneous vasculitis, erythema multiforme, Stevens-Johnson syndrome, Toxic epidermal necrolysis, Guillain-Barre syndrome, and neuropathies. Reactivation of tuberculosis and hepatitis B virus has been reported in psoriasis trials. Fatal infections have occurred in patients who 
Journal of College of Medical Sciences-Nepal,2011,Vol-7,No-1

have been on other immunosuppressive medications in addition to infliximab. Increased incidence of hepatosplenic T-cell lymphoma have been reported. ${ }^{13}$

\section{Conclusion}

Infliximab is a proven powerful agent that is an asset to psoriasis patients as a monotherapy. It has the highest efficacy after 12 weeks of therapy. Infliximab provide significant QoL improvement, like any medication. However, there is a risk of rare severe adverse events. ${ }^{12}$ Infliximab requires intravenous infusion approximately every 8 weeks and patients enjoy the freedom from therapy.

Once again, it should be restated that Psoriasis is typically a chronic disease and there is a need for safe long-term therapies.

\section{References}

1. E. Christophers. Psoriasis - epidemiology and clinical spectrum. Clin. Exp. Dermatol 2001; 26: 314-20.

2. L. Lundberg, M. Johannesson, M. Silverdahl et al. Health-related quality of life in patients with psoriasis and atopic dermatitis measured with SF-36, DLQI and a subjective measure of disease activity. Acta Derm. Venereol 2000; 80: 430-4.

3. S. R. Rapp, S. R. Feldman, M. L. Exum et al. Psoriasis causes as much disability as other major medical diseases. J. Am. Acad. Dermatol 1999; 41: 401-7.

4. S. R. Feldman, R. Garton, W. Averett et al. Strategy to manage the treatment of severe psoriasis: considerations of efficacy, safety and cost. Expert Opin. Pharmacother 2003; 4: 1525-33.

5. R. S. Stern. Inpatient hospital care for psoriasis: a vanishing practice in the United States. J. Am. Acad. Dermatol 2003; 49: 445.
6. J. Koo. Systemic sequential therapy of psoriasis: a new paradigm for improved therapeutic results. J. Am. Acad. Dermatol 1999; 41: S25-S28.

7. S. Feldman. Advances in psoriasis treatment. Dermatol. Online J 2000; 6: 4.

8. K. B. Gordon, R. G. Langley, C. Leonardi et al. Clinical response to adalimumab treatment in patients with moderate to severe psoriasis: double-blind, randomized controlled trial and open-label extension study. J. Am. Acad. Dermatol 2006; 55: 598-606.

9. B. J. Scallon, M. A. Moore, H. Trinh et al. Chimeric anti-TNF-á monoclonal antibody cA2 binds recombinant transmembrane TNF-á and activates immune effector functions. Cytokine 1995; 7: 251-9.

10. A. B. Gottlieb, R. Evans, S. Li et al. Infliximab induction therapy for patients with severe plaque-type psoriasis: a randomized, double-blind, placebo-controlled trial. J. Am. Acad. Dermatol 2004; 51: 534-42.

11. B. Haraoui, L. Cameron, M. Ouellet et al. Antiinfliximab antibodies in patients with rheumatoid arthritis who require higher doses of infliximab to achieve or maintain a clinical response. J. Rheumatol 2006; 33: 31-6.

12. S. R. Feldman, K. B. Gordon, M. Bala et al. Infliximab treatment results in significant improvement in the quality of life of patients with severe psoriasis: a double-blind placebo-controlled trial. Br. J. Dermatol 2005; 152: 954-60.

13. R. A. Hansen, G. Gartlehner, G. E. Powell et al. Serious adverse events with infliximab: analysis of spontaneously reported adverse events. Clin. Gastroenterol. Hepatol 2007; 5: 729-35. 